\title{
Wnt signalling in the gut microbiota-bone axis
}

The probiotic strain Lactobacillus rhamnosus GG (LGG) can promote bone formation and increase bone density in mice. New findings published in Immunity provide insights into this gut microbiotabone pathway, which might have therapeutic implications for diseases such as osteoporosis.

According to the new findings, LGG-induced upregulation of the gut metabolite butyrate expands the regulatory $\mathrm{T}\left(\mathrm{T}_{\mathrm{reg}}\right)$ cell pool, which subsequently increases the production of the osteogenic Wnt ligand Wnt10b by $\mathrm{CD}^{+} \mathrm{T}$ cells to stimulate bone formation.

The researchers found that, as expected, dietary supplementation with LGG resulted in increased bone formation and bone mass in young mice. In the gut, LGG indirectly stimulated the production of butyrate by expanding strains of butyrate-producing bacteria.

Butyrate is known to promote the expansion of peripheral $\mathrm{T}_{\text {reg }}$ cells, and indeed in the new study LGG supplementation increased the numbers of $\mathrm{T}_{\text {reg }}$ cells in the gut and bone marrow. Further experiments using butyrate supplementation and $\mathrm{T}_{\text {reg }}$ cell depletion indicated that LGG affects bone mass through a butyrate-dependent $\mathrm{T}_{\text {reg }}$ cell-mediated pathway.

Both LGG and butyrate supplementation increased the expression of Wnt10b in the bone marrow, which was attributed to an increase in expression by $\mathrm{CD} 8^{+}$ $\mathrm{T}$ cells. Further analysis revealed the pivotal role of Wnt10b in the LGG-bone pathway, and suggested that butyrate indirectly increases CD8 ${ }^{+} \mathrm{T}$ cell expression of Wnt10b via $\mathrm{T}_{\text {reg }}$ cells. Butyrate treatment resulted

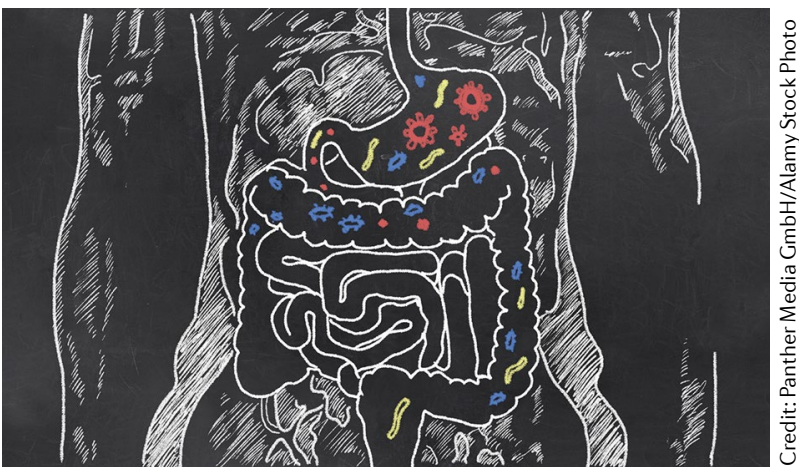

in a $\mathrm{T}_{\text {reg }}$-cell dependent increase in binding of the transcription factors NFAT1 and SMAD3 to the Wnt $10 b$ promoter in $\mathrm{CD}^{+} \mathrm{T}$ cells, promoting Wnt10b expression.

"Butyrate may, therefore, be used [as an] alternative to probiotics and may represent a novel treatment for osteoporosis," explains Roberto Pacifici, corresponding author of the study. "There is a need for a butyrate

indirectly

increases

$\mathrm{CD}^{+} \mathrm{T}$ cell

expression of

Wnt10b via

$\mathrm{T}_{\text {reg }}$ cells clinical trial with LGG or butyrate to determine if these substances prevent bone loss and improve skeletal development."

Jessica McHugh

ORIGINAL ARTICLE Tyagi, A. M. et al. The microbial metabolite butyrate stimulates bone formation via T regulatory cell-mediated regulation of WNT10B expression. Immunity https://doi.org/10.1016/j.immuni.2018.10.013 (2018)

\section{TARGETED THERAPIES}

\section{Preventing immune- complex-mediated disease}

Immune-complex deposition and Fc $\gamma$ receptor $(F c \gamma R)$-mediated activation of inflammatory responses by such immune complexes is a central pathogenic process in a variety of autoimmune diseases, including systemic lupus erythematosus (SLE), rheumatoid arthritis (RA) and vasculitis. A new study reports the development of a humanized anti-Fc $\gamma$ RIIA antibody (VIB9600) that might function as a broad-spectrum therapy for these and other diseases.

"Although it has been known for some time that targeting F $_{c} \gamma$ RIIA is a potential therapeutic strategy for these autoimmune diseases, difficulties relating to the development of an effective and specific antibody have prevented clinical translation," notes Bo Chen, a corresponding author of the new study.

The researchers now show that VIB9600 can inhibit immune-complex responses in a variety of contexts, but importantly without also targeting the highly homologous (but inhibitory) Fc $\gamma$ RIIB and without activating antibody-dependent or complement-dependent cytotoxicity.

VIB9600 inhibited immune-complexinduced production of type 1 interferon by plasmacytoid dendritic cells, and TNF and IL- 6 by monocytes, which are inflammatory pathways known to be central to SLE and RA, respectively.

VIB9600 also inhibited autoantibody-induced production of reactive oxygen species by neutrophils, a pathway known to contribute to vasculitis pathogenesis.
The researchers also showed that, aside from blocking its target Fc receptor, VIB9600 can reduce expression of Fc $\gamma$ RIIA by neutrophils and platelets in Fc $\gamma$ RIIA-transgenic mice. Consequently, these mice were resistant to thrombocytopenia as well as to neutrophil-mediated nephritis and arthritis, in part owing to a reduction in immune-complex signalling.

Finally, the researchers showed that the antibody meets clinical expectations for pharmacokinetics and pharmacodynamics when injected into cynomolgus monkeys, and no adverse events were recorded during a 3-month multiple-dose GLP toxicology study and a subsequent 8-week follow-up period. The pharmacology and safety of VIB9600 are now under investigation in humans in a phase I clinical trial conducted by Viela Bio.

Nicholas J. Bernard

ORIGINAL ARTICLE Chen, B. et al. Humanised effector-null Fc $\gamma R$ IIIA antibody inhibits immune complex-mediated proinflammatory responses. Ann. Rheum. Dis. https://doi.org/10.1136/ annrheumdis-2018-213523 (2018) 